\title{
Chronic Lymphocytic Leukaemia in 2020: the Future Has Arrived
}

\author{
Kate Milne ${ }^{1,2} \cdot$ Beattie Sturrock $^{1,2} \cdot$ Timothy Chevassut ${ }^{1,2}$
}

Published online: 14 March 2020

(C) The Author(s) 2020

\begin{abstract}
Purpose of Review Chronic lymphocytic leukaemia is now recognised as a heterogenous disease with a variety of clinical outcomes. Here we summarise the way it is currently stratified according to genetic risk and patient characteristics and the treatment approaches used for these different subgroups.

Recent Findings Certain patients appear to sustain MRD negativity after combination chemoimmunotherapy, leading to the suggestion that their CLL may be cured. However, 17p-deleted, p53-mutated or IGHV-UM subgroups are generally resistant to FCR, and much better responses are seen with ibrutinib and venetoclax, frequently inducing MRD negativity that hopefully will be translated into durable remissions.

Summary Small molecule inhibitors have already revolutionised CLL treatment. Going forward, we anticipate their use in the majority of patients, early after diagnosis and with curative intent.
\end{abstract}

Keywords Chronic lymphocytic leukaemia $\cdot$ Chemoimmunotherapy $\cdot$ Ibrutinib $\cdot$ Venetoclax $\cdot$ Future

\section{Introduction}

Chronic lymphocytic leukaemia (CLL) is the commonest leukaemia in the world, with 4.9 new diagnosis per 100,000 per year in the UK and USA. The malignant clonal proliferation and accumulation of mature B-lymphocytes is predominantly identified in older patients with a median age of 74 at diagnosis $[1,2]$. The majority of patients are monitored with a "watch and wait approach' until the balance of risks and benefits favours treatment initiation. In some cases, treatment may never be needed whilst in others, the disease is more aggressive with rapid progression and death from disease-related causes a few years after diagnosis [3]. This disparity in

This article is part of the Topical Collection on Leukemia

Kate Milne

kate.milne5@nhs.net

Beattie Sturrock

Beattie.sturrock@nhs.net

Timothy Chevassut

T.Chevassut@bsms.ac.uk

1 Brighton and Sussex Medical School, University of Sussex, Brighton BN1 9PS, UK

2 Royal Sussex County Hospital, Eastern Road, Brighton BN2 5BE, UK outcome highlights the heterogeneity of CLL and the importance of risk stratification to guide treatment decisions. unlike, its myeloid counterpart - chronic myeloid leukaemia - a pathognomonic driving mutation, BCR-ABL, has not been identified, and this slightly delayed the development of targeted therapies. However, over the last two decades, a dramatic increase in our understanding of the pathogenesis of the disease has led to the development of small molecule inhibitors for CLL targeting the B cell receptor pathway and the apoptotic regulator BCL2. Some of these newer therapies appear to be so effective; they may provide a curative option for patients, previously treatment aimed to establish disease control. In this review, we will briefly discuss recent advances in our understanding of the molecular pathology of CLL and then, in more detail, the way that CLL is managed in the UK.

\section{New Developments in the Understanding of the Pathogenies of CLL}

Our understanding of the genetics of CLL and the implications on patient outcome began with the publication by Doner et al. in 2000 which identified four recurrent genetic lesions by fluorescence in situ hybridisation (FISH) and showing that their presence predicted disease prognosis. It was found that 
a lesion at $17 \mathrm{p} 13$ and $11 \mathrm{q} 23$ indicated a poor prognosis compared with 13q14 and 12q; this formed the basis of the FISH probe set used routinely during CLL diagnostics [4]. The utility of FISH is limited by the need to identify particular region to probe and by the number of probes that can be used at one time. Unlike FISH, G-banding karyotyping which can be applied to all the chromosomes simultaneously, required cells to be in metaphase, various compounds have been used to raise the yield of metaphase cells in CLL - the combination of DSp30/IL2 has shown to do so without inducing artefactual chromosomal aberrations [5, 6]. Using this technique, it has been demonstrated $16-19 \%$ of patients have a complex karyotype ( $3>$ abnormalities) that this predicts poor outcome independent of P53 status, and the presence of 5 of more is associated with a P53 mutations and perhaps due to this association, a more aggressive course [7]. These techniques have been replaced in research by chromosomal micro arrays and single nucleotide polymorphisms arrays which do not require the cells to be in the cell cycle and whilst the concordance of these approaches is reported in small studies, their incorporation into large trials is awaited [8].

CLL patients can be categorised into two groups depending on the mutational status, established by PCR or next generation sequencing, of the variable region immunoglobulin heavy chain (IGHV) when compared with germline sequence. During the process of VDJ rearrangement and somatic hypermutation of B cells occurs within the germinal centres generating receptors capable of recognising an extensive range of antigens. IGHV-mutated (IGHV-M) CLL, with a mutation status of $>2 \%$, compared with germline sequence as an immunophenotype and gene expression profile similar to that of post GC CD27+, T cell dependent memory B cells. Whilst IGHV-unmutated (IGHV-UM) CLL cells adopted a phenotype gene expression profile and epigenome that resemble a GC-naïve CD27- B cell $[9,10]$. This difference is reinforced when comparing the BCRs of the two subtypes with IGHVUM carrying low affinity, poly-reactive and self-reactive $\mathrm{BCR}$, and IGHV-M has higher affinity, olio or mono reactive receptors [11]. This may contribute to the poorer prognosis seen with IGHV-UM CLL $[12,13]$.

Next generation sequencing confirmed that the mutational rate in CLL is similar to that of other haematological cancers and lower than solid malignancy, with each patients having a small number of recurrently mutated driver genes - unsurprisingly, many of the mutated genes identified in these studies are involved in cell replication, DNA repair apoptosis and signalling [14-17]. The subtypes, the 4 chromosomal abnormalities discussed earlier have also been shown to be associated with different recurrent gene mutations, similarly to IGHV status, which may explain the heterogeneity in there clinical course. For example, $17 \mathrm{p} 13$ deletion occurs with mutation of the remaining p53 resulting in homozygous inactivity and SF3B1, a protein involved in the regulation of the spliceosome with 11q deletion $[18,19]$. In these studies, a quarter of patients had a mutation in a gene involved in RNA splicing or repair-a possible avenue for new drug development [20].

As well as identification of mutated genes in CLL, the mechanisms of gene expression regulation have also been studied. It is known that BCL2 an antiapoptotic protein is overexpressed in CLL and that its expression increases with chemotherapy [21-23]. BCL2 combines with $\mathrm{BH} 3$ and binds to BIM, preventing BIM triggering apoptosis [24]. The recurrent deletion of $13 \mathrm{q} 14$ is seen in $50 \%$ of de novo CLL; it has been shown that this deletion results in the loss of expression of micro RNA (miRNA) 15-A/16-1 [25, 26]. As miRNA 15$A$ and 16-1 normal interfere in the transcription of BCL2, their loss contributes to its overexpression in CLL [27].

Interaction with other cells in the microenvironment is crucial to the survival and replication of CLL cells, as shown by the rapid apoptosis of CLL cells in vitro and the reduction in this when co-cultured with non-tumoral bystander cells [11]. The lymph nodes can be the key site of CLL proliferation, with higher activation of the NFKb and the BCR signalling pathways crucial for this process than the bone marrow of peripheral blood [28], whilst the bone marrow creates a protective niches preventing spontaneous and drug-induced apoptosis cells $[29,30]$. Interruption of these interactions may force the cells into the peripheral circulation, therefore increasing their susceptibility to drug-induced chemotherapy.

\section{Treatment of Chronic Lymphocytic Leukaemia in 2019}

Most patients with CLL present with an incidental finding of a lymphocytosis, they may have palpable lymphadenopathy or organomegaly, bone marrow involvement can lead to anaemia and thrombocytopenia. Occasionally, patients may have the constitutional B symptoms with an unexplained fever of over $38^{\circ}$, weight loss of $>10 \%$ in less than 6 months and night sweats. A complication of CLL such as a high-grade disease transformation, an autoimmune disease or a severe infection may lead to its diagnosis [31].

The following diagnostic criteria are stipulated by the international workshop of CLL (iwCLL) and reiterated in the WHO classification of lymphoid neoplasms. The lymphocytes count in the peripheral blood must be greater than $5 \times$ $10 \times 9$, persistent for 3 months, and clonality of this population must be shown by flow cytometry for light chain restriction, CD5, CD23, CD79b and surface immunoglobulin expression with low levels of CD20. The presence of smudge/ smear cells, an artefact of blood film production, is a typical finding in CLL [32]. If the WCC is less than $5 \times 10 / \mathrm{L}$ with no other signs of lymphoproliferative disorder, this constitutes monoclonal B cell lymphocytosis which is thought as a precursor to CLL, with a rate of progression of $1 \%$ per year [33]. 
Identification of a clonal population of mature B cells within the lymph nodes or extranodal tissues without a peripheral blood lymphocytosis is referred to as a small lymphocytic lymphoma [34]. Fluorescence in situ hybridisation is used to identify chromosomal rearrangements which can differentiate CLL from other conditions such as mantle cell lymphoma as well as helping with disease stratification.

\section{When to Initiate Treatment}

In addition to diagnostic criteria, the iwCLL guidelines dictate when to initiate treatment, based on patients' symptoms, full blood counts and physical examination. The presence of constitutional symptoms, progressive lymphocytosis, a doubling time of less than 6 months, an $\mathrm{Hb}$ of less than $100 \mathrm{~g} / \mathrm{L}$ or a platelet count of less than $100 \times 10^{9} / \mathrm{L}$ as well as progressive or symptomatic bulky lymphadenopathy/organomegaly or treatment-resistant autoimmune thrombocytopaenia or anaemia are indications to start treatment [32]. In some circumstances, treatment may be initiated at diagnosis, but it is much more common for patient to be monitored for signs of increasing disease activity often over many years.

\section{Patient Risk Stratification}

Similar to the management of most malignancy, the most suitable treatment for a patient with CLL is selected based on genetic features of the disease itself- the presence of a P53 mutation or $17 \mathrm{p}$ deletion and the mutational state of the IGHV - and patient factors [35]. The algorithm for treatment selection followed by haematologists in the UK is shown in Fig. 1. Disruption of P53, a tumour suppressor crucial in many cancers, is known to result in very poor response to combination immunochemotherapy, and therefore, patients are treated with molecular therapies first line [18]. If the IGHV is unmutated, compared with the germline sequence, this confers a poor survival risk when compared with patient with a mutated IGHV [12, 13]. The co-morbidities and performance status of individual patients are crucial in setting treatment goals and treatment selection. Treatment success or disease progression is determined on similar criteria to treatment initiation-repeat CT scans are not recommended out with clinical trials. The identification of minimal residual disease (MRD) on peripheral blood or bone marrow aspirate by flow cytometry, with a sensitivity of $0.01 \%$, is becoming increasingly important as MRD negativity after chemoimmunotherapy is associated with prolonged progression-free and overall survival - in the future, early evidence of MRD negativity may allow for shorter treatment regimens [36, 37].
Upon treatment failure on combination chemoimmunotherapy or ibrutinib, there are now several available options for relapsed disease including the PI3K inhibitor idelalisib and the BCL2 inhibitor Venetoclax, also shown in Fig. 1.

\section{Combination \\ Immunochemotherapy_Fludarabine, Cyclophosphamide, Rituximab (FCR) and Other CD20 Antibodies}

Young fit patients, with a creatine clearance of greater than 70 and a comorbidity scale of 6 or less without P53 mutations and with mutated IGHV, are treated with combination chemoimmunotherapy, fludarabine, cyclophosphamide and rituximab (FCR) $[38,39]$. Prior to the introduction of immunotherapy chlorambucil (CLB), an alkylating agent or fludarabine, a purine analogue monotherapy was the mainstay of treatment - the combination of these agents did not improve response rates but did carry much higher haematological toxicity [40]. The addition of cyclophosphamide to fludarabine (FC) led to an improved progression-free survival (PFS) and overall survival (OS) without an increase in serious adverse events compared with fludarabine monotherapy [41]. The approval of rituximab - a monoclonal antibody targeting CD20, a glycosylated cell surface protein expressed on mature B cells - provided a treatment option for many B cell malignancies. In the CLL8 trial, patients were randomised to receive either FC or FCR, which gave a response rate of 85 and $92 \%$ respectively. Particular subgroups of patients appear to have particularly good long-term outcomes, those with a mutated IGVH, del(13q), trisomy 12 or del(11q) or MRD negative remission $[42,43 \bullet]$. The durability of these remissions led to the suggestion that FCR may be curative for some patients. There is emerging evidence that reduced doses or 3 rather than 6 cycles result in non-inferior PFS and OS with a lower burden of cumulative toxicity [36].

Whilst FCR is known to result in longer PFS compared with bendamustine and rituximab (BR) in younger patients, the benefit was not seen in patients over 65 - and a lower rate of serious infections were seen in the BR cohort. Therefore, in patients over 65 who do not qualify for ibrutinib BR tended to be favoured, unless there is a contraindication to bendamustine when chlorambucil rituximab could be used accepting that this had a poorer response rate [44, 45]. The CLL11 trial looked specifically at the treatment of older patients with co-morbidities and the impact of a new anti CD20 monoclonal antibody, obinutuzumab. Treatment with obinutuzumab-CLB compared with R-CLB and CLB monthotherapy increased response rates and prolonged PFS (median PFS, 26.7 months with obinutuzumab-CLB vs 11.1 months with CLB alone; 16.3 months with R-CLB) 
Fig. 1 Flow chart showing the decision process and treatment options in management of previously untreated CLL that meets the iwCLL criteria for treatment and in relapsed or refractory disease

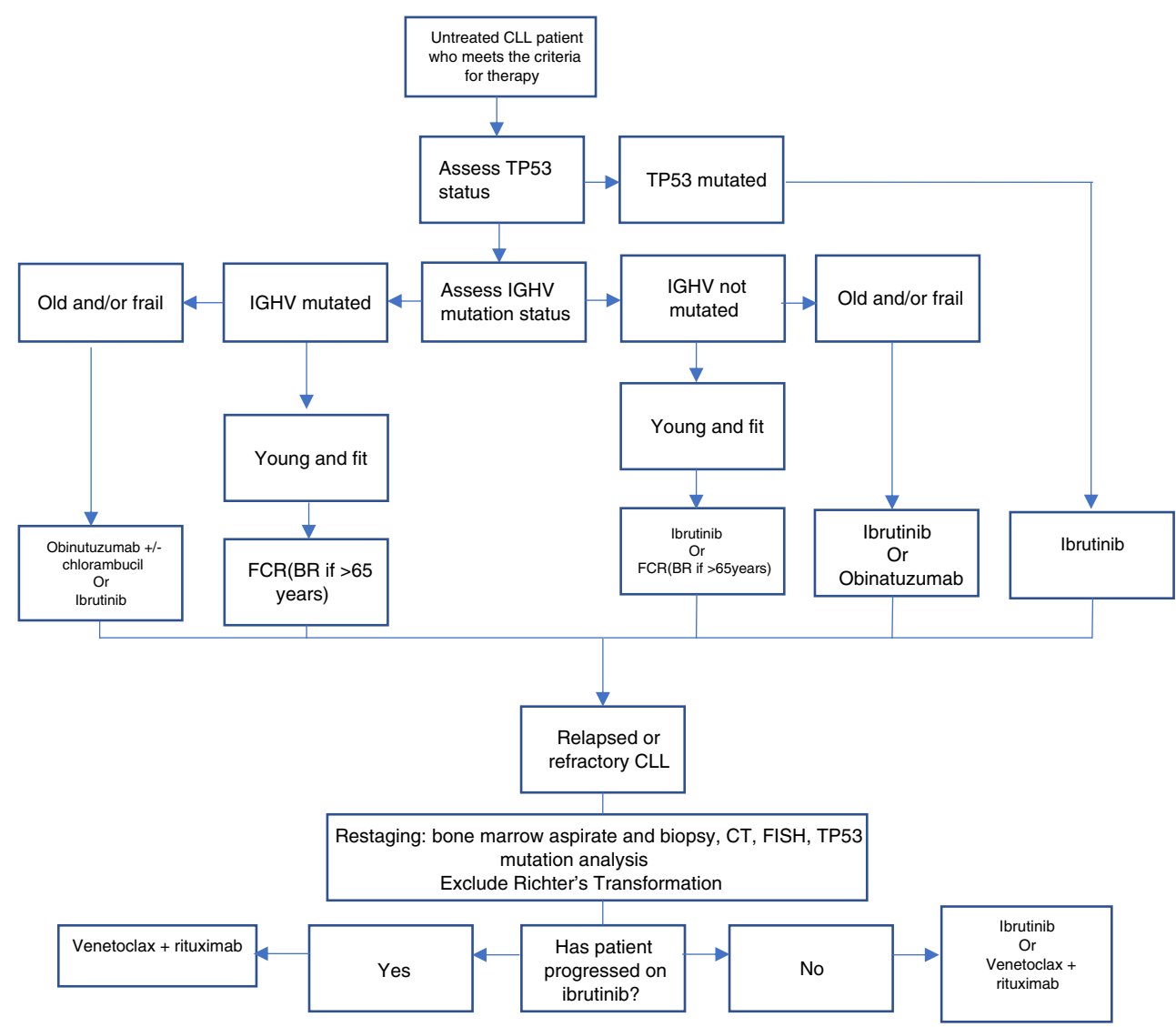

[46]. Currently, results of a large clinical trial multicentre trial, with over 900 participants are awaited, comparing singleagent obinutuzumab and its combination with FC, chlorambucil or bendamustine in untreated and relapsed CLL is awaited; it is possible that obinutuzumab will replace rituximab in the long-standing FCR regime [47, 48].

\section{Bruton Tyrosine Kinase Inhibitors-lbrutinib}

The B cell receptor and downstream signalling pathways are crucial to the survival and proliferation of malignant cells in CLL. Unlike their healthy counterparts in CLL, signalling from the B cell receptor is activated independently of appropriate antigen stimulation - autologous activation. Bruton tyrosine kinase (BTK) which is only slightly downstream of the receptor itself activates the cell survival pathway NfK-B and MAP kinases, its inhibition leads to apoptosis of CLL cells [49]. Ibrutinib is an orally available small molecular inhibitor that binds to BTK preventing its kinase activity. This affects multiple signalling pathways and disrupts the interactions between CLL cells and the microenvironment leading to further apoptosis.

Initially trialled in relapsed or refractory CLL, response rates to ibrutinib were between 84 and $97 \%$ and complete response rates were $12-23 \%$ [50]. This was independent of previously identified poor prognostic factors including advanced stage disease, number of previous lines of treatment and del 17(p). The RESONATE trial, a multicentre phase 3 open-label trial with 391 participants, compared ibrutinib with an anti-CD20 antibody, ofatumumab, note no longer in use, in a cohort of patients with relapsed CLL/SLL. Ibrutinib significantly improved the response rate, PFS and OS. The median PFS of 8.1 months in the ofatumumab group whilst at 9.4 months, the median PFS had not been reached in the ibrutinib group, PFS of $88 \%$ at 6 months [51]. RESONATE 2 showed that ibrutinib was more effective than single-agent chlorambucil as a first-line treatment in patients aged over 65 . Ibrutinib significantly prolonged overall survival; estimated survival rate at 24 months was $98 \%$ with ibrutinib and $85 \%$ with chlorambucil, with a relative risk of death that was $84 \%$ lower in the ibrutinib group. The overall response rate was higher with ibrutinib than with chlorambucil ( $86 \%$ vs $35 \%$ ) $[51,52]$. It is important to note that unlike RESONATE in RESONATE 2 , the presence of a $17 \mathrm{p}$ deletion was an exclusion criterion, and the use of single-agent chlorambucil without a CD20 antibody is now very rare. A more relevant comparison of ibrutinib versus bendamustine and rituximab in patients over 65 did show ibrutinib to confer a significant benefit. The estimated percentage of patients with progression-free survival at 2 years was $74 \%$ with bendamustine plus rituximab and $87 \%$ with ibrutinib alone. 
Combined rituximab and ibrutinib provided no additional benefit compared with ibrutinib alone with an estimated PFS of $88 \%$ at 2 years [53]. Ibrutinib and obinutuzumab does appear to be beneficial compared with CLB-obinutuzumab with a high estimated 30-month overall survival and fewer serious adverse events in the iLLUMINATE phase 3 trial of first-line treatment [54] (Table 1).

The use of ibrutinib first line in younger patients, without $17 \mathrm{p}$ deletion or $\mathrm{p} 53$ mutation, has not yet been clearly demonstrated, one trial has reported an improvement in PFS and OS with ibrutinib and rituximab compared with FCR as a firstline treatment of CLL in patients under 70 [55]. However, as of yet, there has only been a short follow-up period, and there was a surprisingly high number of deaths in the FCR arm indicating further work is needed.

The results of the RESONATE trials have not been replicated in the clinic; the UK ibrutinib real world study reported that $44 \%$ of the patients had a dose reduction, interruption of complete cessation in the first 12 months compared with $4 \%$ in the resonate study. The OS at 12 months was $83 \%, 89 \%$ for patients with no dose reduction or cessation of less than 14 days compared with $90 \%$ in the RESONATE [56 ${ }^{\circ}$. A theoretical benefit of small molecule inhibitors is the reduced side effect profile but due to off-target effects, these are still not negligible with significant bleeding, recurrent infections and cardiac toxicity, particular atrial fibrillation, being the most common reasons for treatment interruption or cessation.

We are now beginning to understand the mechanisms underlying the ibrutinib failure to ibrutinib failure. Comparisons of targeted deep sequencing before initiation of ibrutinib and at the point or either CLL progression or Richter's transformation identified new mutations in BTK or PLG2 that were not present prior to treatment [57]. A larger prospective study also identified these mutations in some patients who had not yet shown signs of clinical relapse suggesting sequencing may become an indicator of when further intervention is required [58].

\section{Phosphatidylinositol 3-Kinases(PI3K) Inhibitors-Idelalisib}

The PI3K signalling pathway, downstream of the B cell receptor, is constitutively activated in CLL and is required for their survival and proliferation $[59,60]$. Idelalisib is a potent and specific inhibitor of PI3K isoform expression of which is restricted to cells of haematopoietic origin. Idelalisib induces apoptosis in CLL cells whilst T cells and NK cell are unaffected. Like ibrutinib, idelalisib has multiple mechanisms of action, such as disruption of the CLL cell CXC12 and CXC13 driven chemotaxis towards stromal cells and their migration beneath them; this may keep the cells within the peripheral

Table 1 Significant randomised and more recent phase $1 / 2$ trials using targeted small molecular inhibitors in CLL

\begin{tabular}{|c|c|c|c|c|c|c|c|}
\hline Treatment & $\begin{array}{l}\mathrm{FL} / \\
\mathrm{RR}\end{array}$ & Number & Age & ORR & NMRD & $2 \mathrm{YOS}$ & Reference \\
\hline \multicolumn{8}{|l|}{ Randomised phase 3 trials } \\
\hline Ibrutinib & $\mathrm{RR}$ & 195 & 67 & $63 \%$ & NR & 1 year $90 \%$ & Byrd 2014 [51] \\
\hline Ofatumumab & & 196 & 67 & $4 \%$ & NR & 1 year $81 \%$ & \\
\hline Ibrutinib & FL & 136 & 73 & $86 \%$ & NR & $98 \%$ & Burger 2015 [52] \\
\hline Chorambucil & & 133 & 72 & $35 \%$ & NR & $85 \%$ & \\
\hline Ibrutinib & $\mathrm{FL}$ & 182 & 71 & $93 \%$ & $1 \%$ & $90 \%$ & Woyach 2018 [53] \\
\hline Ibrutinib/rituximab & & 182 & 71 & $94 \%$ & $4 \%$ & $94 \%$ & \\
\hline Ibrutinib/obinutuzumab & FL & 113 & 70 & $88 \%$ & $35 \%$ & $30 \mathrm{~m} 86 \%$ & Moreno 2019 [54] \\
\hline CLB/obinutuzumab & & 116 & 72 & $73 \%$ & $25 \%$ & $30 \mathrm{~m} 85 \%$ & \\
\hline Bendamustine/rituximab & & 183 & 70 & $81 \%$ & $8 \%$ & $95 \%$ & \\
\hline Ibrutinib/rituximab & FL & 354 & 58 & $96 \%$ & $8 \%$ & 3 years $99 \%$ & Shanfelt 2019 [55] \\
\hline FCR & & 175 & 57 & $81 \%$ & $59 \%$ & 3 years $92 \%$ & \\
\hline Idelalisib/rituximab & $\mathrm{RR}$ & 110 & 71 & $81 \%$ & NR & 1 year $92 \%$ & Furman 2014 [62] \\
\hline Rituximab & & 110 & 71 & $13 \%$ & NR & 1 year $80 \%$ & \\
\hline Venetoclax/rituximab & $\mathrm{RR}$ & 194 & 65 & $92 \%$ & $62 \%$ & $92 \%$ & Seymour 2018 [68] \\
\hline Bendamustine/rituximab & & 195 & 65 & $72 \%$ & $13 \%$ & $87 \%$ & \\
\hline \multicolumn{8}{|l|}{ Phase $1 / 2$ trials } \\
\hline Ibrutinib/venetoclax & FL & 80 & 65 & $88 \%$ & $61 \%$ & 1 year $99 \%$ & Jain 2019 [73] \\
\hline Alcalabrutinib & $\mathrm{RR}$ & 61 & 62 & $95 \%$ & NR & 1 year $100 \%$ & Byrd 2016 [75] \\
\hline
\end{tabular}

$F L$ first-line therapy, $R R$ relapsed or refractory, Age median in years, $O R R$ overall response rate, $N M R D$ negative MRD in peripheral blood, 2 YOS 2 -year overall survival rate unless alternate follow-up period specified 
blood increasing their susceptibility to apoptosis induction [60]. Idelalisib was initially evaluated in relapsed and refractory disease including patients with adverse features - bulky lymphadenopathy, $17 \mathrm{p}$ deletion/Tp53 mutation, IGHVunmutated and failure of multiple treatments. Idelalisib had an overall response rate of $72 \%$ in this cohort and PFS of 15.8 months [61]. The combination of idelalisib with rituximab, compared with rituximab alone, leads to higher overall response rate $81 \%$ vs $13 \%$ and a 12 -month survival or $92 \%$ vs $81 \%$. There was also a higher rate of reported serious adverse events in the idelalisib and rituximab group (40\%) - the most common being pneumonia, pyrexia and febrile neutropoenia; it is likely that due to its toxicity, its use will be restricted to relapsed disease [62].

\section{BCL2 Inhibition-Venetoclax}

An ability to evade apoptosis is required for the development of cancer - making its regulatory pathways an important therapeutic target [63]. Venetoclax, a $\mathrm{BH} 3$ mimic, prevents the interaction between BCL2 and BH3 inducing cell death [64]. Earlier BH3 mimetics showed good disease response but induced severe thrombocytopaenia in a phase 1 trial [65]. Venetoclax avoids this due to its higher specificity for BCL2 than some of its predecessors. The phase 1 and 2 trials of venetoclax showed impressive results with an overall response rate of 70-80\% across all prognostic groups including patients with a $17 \mathrm{p}$ deletion/Tp53 mutation. Additionally, unlike the use of ibrutinib and idelalisib, venetoclax-induced MRD negative complete responses [66]. The most important adverse effect was the occurrence of fatal tumour lysis syndrome in the initial phase 1 trial; this could occur after even a single dose of 100 or $200 \mathrm{mg}$. Since the introduction of a strict dosing increment regime, there have been no further deaths attributable to, and a lower incidence of TLS. Monitoring for cytopaenias, infection and hepatotoxicity is also required.

A retrospective analysis of the UK patients started on venetoclax, who had failed a BTK inhibitor and/or a PI3K inhibitor, reported an overall response rate of $88 \%$. At the median follow-up of 15.6 months, the 1-year PFS and OS was $65.0 \%$ and $75.1 \%$, respectively. Of particular interest was the response rate of $80 \%$ in patients who had received both BTK and PI3K inhibitors - a group of patients who previously had no further treatment options other than autologous stem cell transplant [67]. A similar study conducted in the US reported a lower ORR of $72 \%$, but a much higher proportion of patients therapy was held/stopped $29 \%$ compared with $8 \%$ in the UK, and so far, the follow-up period has been significantly shorter-7 months [3]. The use of venetoclax in combination with monthly rituximab had a dramatically improved outcome at 2 years compared with BR in relapsed CLL, and this combination is now commonly used in clinical practice
[68 • ]. Interestingly, the VR regimes result in $62 \%$ of patients having MRD negativity in peripheral blood, compared with $13 \%$ of the BR, hopefully longer follow-up will show that this combination results in a durable remission. Venetoclax is not immune to the development of resistance with identified mechanisms being mutation of BCL2 or the compensatory over expression of MCL1, another BCL family member [69, 70]. However with the promising MRD negativity seen in VR regimes, it is hoped that combination therapy that includes venetoclax may prevent the development of resistance and treatment failure.

As the apoptotic pathways are targeted by venetoclax are universal, there is hope its success in CLL will be replicated. Within haematology, there have been promising results supporting the use of venetoclax in combination with hypomethylating agents such as daunorubicin in relapsed AML or with cytidine analogues as a first-line treatment for AML in older patients [71].

\section{Conclusion—the Future of CLL Treatment}

The last decade has seen dramatic change in CLL treatment but there are important trial results awaited in 2020 and further into the future. The watch and wait principle of CLLdelaying treatment initiation until a progression means the iwCLL criteria are met is from the chemoimmunotherapy era. Several trials challenging this with the initiation of ibrutinib at diagnosis in patients without an indication to treat but high-risk disease are active, with results due to be reported in mid-2020 or in ongoing recruitment [72, 73]. Additionally, longer term follow-up in patients treated with small molecule inhibitors is required to see if the achievement of MRD negativity improves survival as seen with FCR-it is likely that monitoring MRD will become more common place in CLL, as it is already in other haematological malignancies [74]. The results of a multicentre phase $1 / 2$ trials establishing the maximum tolerated dose of alcalabrutinib - a BTK inhibitor with higher specificity and lower reversibility than ibrutinib-are due to be published in early 2021 indicating that with time, we will likely see an increase in the number of available drugs within the classes of small molecule inhibitors already established [75]. Perhaps, the most exciting current trials in CLL are the use of multiple molecular inhibitors - such as venetoclax and ibrutinib simultaneous - this combination has been shown to provoke a complete remission, including MRD negativity, in patients after a limited duration of treatment meaning that patients may not be committed to indefinite therapy [73*0]. Limiting length of treatment regimens has the advantageous of reduced exposure, and therefore, toxicity particularly relevant in CLL due to the increasingly complex comorbidities seen in this patient cohort. It is recommend that fit patients with relapsed CLL, or a $17 \mathrm{p}$ deletion, should be 
considered for allogeneic transplantation after the failure of one kinase inhibitor - whilst venetoclax provides a valid option for these patients in the future CAR T cells may also prevent the need for transplant [76]. Small studies of antiCD19 CAR T cells in patients who relapsed on BTK inhibitors have shown response rates of over $70 \%$ and a survival rate of $100 \%$, although only after 6 months of follow-up. Further larger trials are required, but we can be cautiously optimistic; this may provide a safer alternative to transplantation in patients who fail small molecule therapy $[77,78]$.

Funding Information Dr. Milne is funded by a National institute for Health Research (NIHR) Academic Clinical Fellowship award. Dr. Chevassut is supported by a project grant from Bloodwise, UK.

\section{Compliance with Ethical Standards}

Conflict of Interest The authors declare that they have no conflict of interest.

Human and Animal Rights and Informed Consent This article does not contain any studies with human or animal subjects performed by any of the authors.

Open Access This article is licensed under a Creative Commons Attribution 4.0 International License, which permits use, sharing, adaptation, distribution and reproduction in any medium or format, as long as you give appropriate credit to the original author(s) and the source, provide a link to the Creative Commons licence, and indicate if changes were made. The images or other third party material in this article are included in the article's Creative Commons licence, unless indicated otherwise in a credit line to the material. If material is not included in the article's Creative Commons licence and your intended use is not permitted by statutory regulation or exceeds the permitted use, you will need to obtain permission directly from the copyright holder. To view a copy of this licence, visit http://creativecommons.org/licenses/by/4.0/.

\section{References}

Papers of particular interest, published recently, have been highlighted as:

- Of importance

•- Of major importance

1. Chronic lymphocytic leukemia - Cancer Stat Facts [Internet]. [cited 2019 Nov 17]. Available from: https://seer.cancer.gov/statfacts/ $\mathrm{html} / \mathrm{clyl} . \mathrm{html}$.

2. Chronic lymphocytic leukaemia (CLL) statistics $\mid$ Cancer Research UK [Internet]. [cited 2019 Nov 17]. Available from: https://www. cancerresearchuk.org/health-professional/cancer-statistics/ statistics-by-cancer-type/leukaemia-cll\#heading-Zero.

3. Mato AR, Thompson M, Allan JN, Brander DM, Pagel JM, Ujjani CS, et al. Real-world outcomes and management strategies for venetoclax-treated chronic lymphocytic leukemia patients in the United States. Haematologica. 2018;103(9):1511-7.
4. Döhner H, Stilgenbauer S, Benner A, Leupolt E, Kröber A, Bullinger L, et al. Genomic aberrations and survival in chronic lymphocytic leukemia. N Engl J Med. 2000;343(26):1910-6.

5. Rai KR, Kipps TJ, Kay NE, Van Dyke D. NIH Public Access. 2011;203(2):134-40

6. Haferlach C, Dicker F, Schnittger S, Kern W, Haferlach T. Comprehensive genetic characterization of CLL: a study on 506 cases analysed with chromosome banding analysis, interphase FISH, IgV H status and immunophenotyping. Leukemia. 2007;21:2442-51.

7. Puiggros A, Collado R, Calasanz MJ, Ortega M, Rivas-delgado A, Luño E, et al. Patients with chronic lymphocytic leukemia and complex karyotype show an adverse outcome even in absence of TP53/ATM FISH deletions. Oncotarget. 2017;8(33):54297-303.

8. Xu X, Johnson EB, Leverton L, Arthur A, Watson Q, Chang FL, et al. The advantage of using SNP array in clinical testing for hematological malignancies - a comparative study of three genetic testing methods. Cancer Genet [Internet]. 2013 Sep [cited 2019 Nov 17];206(9-10):317-26. Available from: https://linkinghub. elsevier.com/retrieve/pii/S2210776213001245.

9. Seifert M, Sellmann L, Bloehdorn J, Wein F, Stilgenbauer S, Dürig $\mathrm{J}$, et al. Cellular origin and pathophysiology of chronic lymphocytic leukemia. J Exp Med. 2012;209(12):2183-98.

10. Kulis M, Heath S, Bibikova M, Queirós AC, Navarro A, Clot G, et al. Epigenomic analysis detects widespread gene-body DNA hypomethylation in chronic lymphocytic leukemia. Nat Genet. 2012;44(11):1236-42.

11. ten Hacken E, Burger JA. Microenvironment interactions and Bcell receptor signaling in chronic lymphocytic leukemia: implications for disease pathogenesis and treatment. In: Biochimica et Biophysica Acta - Molecular Cell Research, vol. 1863. Amsterdam: Elsevier B.V.; 2016. p. 401-13.

12. Hamblin TJ, Davis Z, Gardiner A, Oscier DG, Stevenson FK. Unmutated $\mathrm{Ig} \mathrm{V}(\mathrm{H})$ genes are associated with a more aggressive form of chronic lymphocytic leukemia. Blood. 1999;94(6):184854.

13. Damle RN, Wasil T, Fais F, Ghiotto F, Valetto A, Allen SL, et al. Ig $\mathrm{V}$ gene mutation status and CD38 expression as novel prognostic indicators in chronic lymphocytic leukemia. Blood. 1999;94(6): 1840-7.

14. Landau DA, Tausch E, Taylor-Weiner AN, Stewart C, Reiter JG, Bahlo J, et al. Mutations driving CLL and their evolution in progression and relapse. Nature. 2015;526(7574):525-30.

15. Puente XS, Pinyol M, Quesada V, Conde L, Ordóñez GR, Villamor $\mathrm{N}$, et al. Whole-genome sequencing identifies recurrent mutations in chronic lymphocytic leukaemia. Nature. 2011;475(7354):101-5.

16. Fabbri G, Rasi S, Rossi D, Trifonov V, Khiabanian H, Ma J, et al. Analysis of the chronic lymphocytic leukemia coding genome: role of NOTCH1 mutational activation. J Exp Med. 2011;208(7):1389401.

17. Guièze R, Robbe P, Clifford R, De Guibert S, Pereira B, Timbs A, et al. Presence of multiple recurrent mutations confers poor trial outcome of relapsed/refractory CLL. Blood. 2015;126(18):2110-7.

18. Rossi D, Gaidano G. The clinical implications of gene mutations in chronic lymphocytic leukaemia. Br J Cancer. 2016;114(8):849-54. https://doi.org/10.1038/bjc.2016.78.

19. Wang L, Lawrence MS, Wan Y, et al. SF3B1 and other novel cancer genes in chronic lymphocytic leukemia. NIH Public Access. 2013;365(26):2497-506.

20. Bonnal S, Vigevani L, Valcárcel J. The spliceosome as a target of novel antitumour drugs. Nat Rev Drug Discov. 2012;11:84759.

21. Hanada M, Delia D, Aiello A, Stadtmauer E, Reed JC. bcl-2 gene hypomethylation and high-level expression in B-cell chronic lymphocytic leukemia. Blood. 1993;82(6):1820-8. 
22. McConkey DJ, Chandra J, Wright S, Plunkett W, McDonnell TJ, Reed JC, et al. Apoptosis sensitivity in chronic lymphocytic leukemia is determined by endogenous endonuclease content and relative expression of BCL-2 and BAX. J Immunol [Internet]. 1996 Apr 1 [cited 2019 Nov 17];156(7):2624-30. Available from: http://www. ncbi.nlm.nih.gov/pubmed/8786328.

23. Pepper C, Thomas A, Hoy T, Bentley P. Chlorambucil resistance in B-cell chronic lymphocytic leukaemia is mediated through failed Bax induction and selection of high Bcl-2-expressing subclones. $\mathrm{Br}$ J Haematol [Internet]. 1999 Mar [cited 2019 Nov 17];104(3):5818. Available from: http://doi.wiley.com/10.1046/j.1365-2141.1999. 01210.x

24. Moore VDG, Brown JR, Certo M, Love TM, Novina CD, Letai A. Chronic lymphocytic leukemia requires BCL2 to sequester prodeath BIM, explaining sensitivity to BCL2 antagonist ABT737. J Clin Invest. 2007;117(1):112-21.

25. Calin GA, Dumitru CD, Shimizu M, Bichi R, Zupo S, Noch E, et al. Frequent deletions and down-regulation of micro- RNA genes miR15 and miR16 at 13q14 in chronic lymphocytic leukemia. Proc Natl Acad Sci U S A. 2002;99(24):13-8.

26. Oscier DG, Gardiner AC, Mould SJ, Glide S, Davis ZA, Ibbotson $\mathrm{RE}$, et al. Multivariate analysis of prognostic factors in CLL: clinical stage, IGVH gene mutational status, and loss or mutation of the p53 gene are independent prognostic factors. Blood. 2002;100(4): 1177-84.

27. Cimmino A, Calin GA, Fabbri M, Iorio MV, Ferracin M, Shimizu $\mathrm{M}$, et al. miR-15 and miR-16 induce apoptosis by targeting BCL2. Proc Natl Acad Sci U S A. 2005;102(39):13944-9.

28. Herishanu Y, Pérez-Galán P, Liu D, Biancotto A, Pittaluga S, Vire $\mathrm{B}$, et al. The lymph node microenvironment promotes B-cell receptor signaling, NF- $\mathrm{kB}$ activation, and tumor proliferation in chronic lymphocytic leukemia. Blood. 2011;117(2):563-74.

29. Kurtova AV, Balakrishnan K, Chen R, Ding W, Schnabl S, Quiroga MP, et al. Diverse marrow stromal cells protect CLL cells from spontaneous and drug-induced apoptosis: development of a reliable and reproducible system to assess stromal cell adhesion-mediated drug resistance. Blood. 2009;114(20):4441-50.

30. Burger JA, Burger M, Kipps TJ. Chronic lymphocytic leukemia B cells express functional CXCR4 chemokine receptors that mediate spontaneous migration beneath bone marrow stromal cells. Blood. 1999;94(11):3658-67.

31. Strati P, Jain N, O’Brien S. Chronic lymphocytic leukemia: diagnosis and treatment. Mayo Clin Proc [Internet]. 2018;93(5):651-64. Available from: https://doi.org/10.1016/j.mayocp.2018.03.002.

32. Hallek M, Cheson BD, Catovsky D, Caligaris-Cappio F, Dighiero G, Döhner H, et al. iwCLL guidelines for diagnosis, indications for treatment, response assessment, and supportive management of CLL. Blood. 2018;131(25):2745-60.

33. Marti GE, Rawstron AC, Ghia P, Hillmen P, Houlston RS, Kay N, et al. Diagnostic criteria for monoclonal B-cell lymphocytosis. Br J Haematol [Internet]. 2005 Aug [cited 2019 Nov 17];130(3):32532. Available from: http://doi.wiley.com/10.1111/j.1365-2141. 2005.05550.x

34. Santos FPS, O'Brien S. Small lymphocytic lymphoma and chronic lymphocytic leukemia: are they the same disease? Cancer J (United States). 2012;18:396-403.

35. Initial treatment of CLL: integrating biology and functional status. PubMed - NCBI [Internet]. [cited 2019 Nov 17]. Available from: https://www.ncbi.nlm.nih.gov/pubmed/26065656.

36. Strati P, Keating MJ, O'Brien SM, Burger J, Ferrajoli A, Jain N, et al. Eradication of bone marrow minimal residual disease may prompt early treatment discontinuation in CLL. Blood. 2014;123(24):3727-32.

37. Böttcher S, Ritgen M, Fischer K, Stilgenbauer S, Busch RM, Fingerle-Rowson G, et al. Minimal residual disease quantification is an independent predictor of progression-free and overall survival in chronic lymphocytic leukemia: a multivariate analysis from the randomized GCLLSG CLL8 trial. J Clin Oncol. 2012;30(9):980-8.

38. Hallek M, Fischer K, Fingerle-Rowson G, Fink AM, Busch R, Mayer J, et al. Addition of rituximab to fludarabine and cyclophosphamide in patients with chronic lymphocytic leukaemia: a randomised, open-label, phase 3 trial. Lancet. 2010;376(9747): 1164-74.

39. Keating MJ, O’Brien S, Albitar M, Lerner S, Plunkett W, Giles F, et al. Early results of a chemoimmunotherapy regimen of fludarabine, cyclophosphamide, and rituximab as initial therapy for chronic lymphocytic leukemia. J Clin Oncol [Internet]. 2005 Jun 20 [cited 2019 Nov 17];23(18):4079-88. Available from: http://www.ncbi.nlm.nih.gov/pubmed/15767648.

40. Hallek M, Eichhorst BF. Chemotherapy combination treatment regimens with fludarabine in chronic lymphocytic leukemia. Hematol J. 2004;5(Suppl. 1).

41. Catovsky D, Richards S, Matutes E, Oscier D, Dyer M, Bezares R, et al. Assessment of fludarabine plus cyclophosphamide for patients with chronic lymphocytic leukaemia (the LRF CLL4 trial): a randomised controlled trial. Lancet. 2007;370(9583):230-9.

42. Rossi D, Terzi-Di-Bergamo L, De Paoli L, Cerri M, Ghilardi G, Chiarenza A, et al. Molecular prediction of durable remission after first-line fludarabinecyclophosphamide-rituximab in chronic lymphocytic leukemia. Blood. American Society of Hematology. 2015;126:1921-4.

43. Fischer K, Bahlo J, Fink AM, Goede V, Herling CD, Cramer P, et al. Long-term remissions after FCR chemoimmunotherapy in previously untreated patients with CLL: updated results of the CLL8 trial. Blood. 2016:208-15 Update on long term outcomes of patients in the CLL8 trial showing that FCR results in lastining remission in patients without 17p deletion of $P 53 \mathrm{mu}-$ tation and IGHV-mutated CLL as well as in patients who achieved MRD negativity in peripheral blood following 6 cycle of FCR.

44. Eichhorst B, Fink AM, Bahlo J, Busch R, Kovacs G, Maurer C, et al. First-line chemoimmunotherapy with bendamustine and rituximab versus fludarabine, cyclophosphamide, and rituximab in patients with advanced chronic lymphocytic leukaemia (CLL10): an international, open-label, randomised, phase 3, non-inferiority trial. Lancet Oncol. 2016;17(7):928-42.

45. Michallet AS, Aktan M, Hiddemann W, Ilhan O, Johansson P, Laribi K, et al. Rituximab plus bendamustine or chlorambucil for chronic lymphocytic leukemia: primary analysis of the randomized, open-label mable study. Haematologica. 2018;103(4):698-706.

46. Goede V, Fischer K, Busch R, Engelke A, Eichhorst B, Wendtner $\mathrm{CM}$, et al. Obinutuzumab plus chlorambucil in patients with CLL and coexisting conditions. N Engl J Med [Internet]. 2014 Mar 20 [cited 2019 Nov 17];370(12):1101-10. Available from: http://www. nejm.org/doi/10.1056/NEJMoa1313984

47. Stilgenbauer S, Leblond V, Foà R, Böttcher S, Ilhan O, Knauf W, et al. Obinutuzumab plus bendamustine in previously untreated patients with CLL: a subgroup analysis of the GREEN study. Leukemia. 2018;32(8):1778-86.

48. A safety and efficacy study of obinutuzumab alone or in combination with chemotherapy in participants with chronic lymphocytic leukemia - Full Text View - ClinicalTrials.gov [Internet]. [cited 2019 Nov 17]. Available from: https://clinicaltrials.gov/ct2/show/ NCT01905943

49. Petlickovski A, Laurenti L, Li X, Marietti S, Chiusolo P, Sica S, et al. Sustained signaling through the B-cell receptor induces Mcl-1 and promotes survival of chronic lymphocytic leukemia B cells. Blood. 2005;105(12):4820-7.

50. Advani RH, Buggy JJ, Sharman JP, Smith SM, Boyd TE, Grant B, et al. Bruton tyrosine kinase inhibitor ibrutinib (PCI-32765) has significant activity in patients with relapsed/refractory B-cell malignancies. J Clin Oncol. 2013;31(1):88-94. 
51. Byrd JC, Brown JR, O’Brien S, Barrientos JC, Kay NE, Reddy $\mathrm{NM}$, et al. Ibrutinib versus ofatumumab in previously treated chronic lymphoid leukemia. N Engl J Med. 2014;371(3):213-23.

52. Burger JA, Tedeschi A, Barr PM, Robak T, Owen C, Ghia P, et al. Ibrutinib as initial therapy for patients with chronic lymphocytic leukemia. N Engl J Med. 2015;373(25):2425-37.

53. Woyach JA, Ruppert AS, Heerema NA, Zhao W, Booth AM, Ding $\mathrm{W}$, et al. Ibrutinib regimens versus chemoimmunotherapy in older patients with untreated CLL. N Engl J Med [Internet]. 2018 Dec 27 [cited 2019 Nov 18];379(26):2517-28. Available from: http://www. nejm.org/doi/10.1056/NEJMoa1812836

54. Moreno C, Greil R, Demirkan F, Tedeschi A, Anz B, Larratt L, et al. Ibrutinib plus obinutuzumab versus chlorambucil plus obinutuzumab in first-line treatment of chronic lymphocytic leukaemia (iLLUMINATE): a multicentre, randomised, open-label, phase 3 trial. Lancet Oncol. 2019;20(1):43-56.

55. Shanafelt TD, Wang XV, Kay NE, Hanson CA, O'Brien S, Barrientos J, et al. Ibrutinib-rituximab or chemoimmunotherapy for chronic lymphocytic leukemia. N Engl J Med. 2019;381(5): 432-43.

56. Leukemia CL. Ibrutinib for relapsed/refractory chronic lymphocytic leukemia: a UK and Ireland analysis of outcomes in 315 patients. Haematologica. 2016;101(12):1563-72 Real world Ibrutinib outcomes in patients in the UK concluding that whilst effective it is much more likely for patients to have the drug held or stopped outwith trial settings.

57. Woyach JA, Furman RR, Liu TM, Ozer HG, Zapatka M, Ruppert AS, et al. Resistance mechanisms for the Bruton's tyrosine kinase inhibitor ibrutinib. N Engl J Med. 2014;370(24):2286-94.

58. Woyach JA, Ruppert AS, Guinn D, Lehman A, Blachly JS, Lozanski A, et al. BTKC481S-mediated resistance to ibrutinib in chronic lymphocytic leukemia. J Clin Oncol. 2017;35(13):143743.

59. Okkenhaug K, Vanhaesebroeck B. PI3K in lymphocyte development, differentiation and activation. Nat Rev Immunol. European Association for Cardio-Thoracic Surgery. 2003;3:317-30.

60. Hoellenriegel J, Meadows SA, Sivina M, Wierda WG, Kantarjian $\mathrm{H}$, Keating MJ, et al. The phosphoinositide 3'-kinase delta inhibitor, CAL-101, inhibits B-cell receptor signaling and chemokine networks in chronic lymphocytic leukemia. Blood. 2011;118(13): 3603-12.

61. Brown JR, Byrd JC, Coutre SE, Benson DM, Flinn IW, WagnerJohnston ND, et al. Idelalisib, an inhibitor of phosphatidylinositol 3-kinase p $110 \delta$, for relapsed/refractory chronic lymphocytic leukemia. Blood. 2014;123(22):3390-7.

62. Furman RR, Sharman JP, Coutre SE, Cheson BD, Pagel JM, Hillmen $\mathrm{P}$, et al. Idelalisib and rituximab in relapsed chronic lymphocytic leukemia. N Engl J Med [Internet]. 2014 Mar 13 [cited 2019 Nov 17];370(11):997-1007. Available from: http://www. nejm.org/doi/10.1056/NEJMoa1315226

63. Hanahan D, Weinberg RA. Leading Edge Review Hallmarks of Cancer: The Next Generation; 2011.

64. Souers AJ, Leverson JD, Boghaert ER, Ackler SL, Catron ND, Chen J, et al. ABT-199, a potent and selective BCL-2 inhibitor, achieves antitumor activity while sparing platelets. Nat Med. 2013;19(2):202-8.

65. Roberts AW, Seymour JF, Brown JR, Wierda WG, Kipps TJ, Khaw SL, et al. Substantial susceptibility of chronic lymphocytic leukemia to BCL2 inhibition: results of a phase I study of navitoclax in patients with relapsed or refractory disease. J Clin Oncol. 2012;30(5):488-96.
66. Stilgenbauer S, Eichhorst B, Schetelig J, Coutre S, Seymour JF, Munir T, et al. Venetoclax in relapsed or refractory chronic lymphocytic leukaemia with $17 \mathrm{p}$ deletion: a multicentre, open-label, phase 2 study. Lancet Oncol. 2016;17(6):768-78.

67. Eyre TA, Kirkwood AA, Gohill S, Follows G, Walewska R, Walter $\mathrm{H}$, et al. Efficacy of venetoclax monotherapy in patients with relapsed chronic lymphocytic leukaemia in the post-BCR inhibitor setting: a UK wide analysis. Br J Haematol. 2019;185(4):656-69.

68.• Seymour JF, Kipps TJ, Eichhorst B, Hillmen P, D’Rozario J, Assouline S, et al. Venetoclax-rituximab in relapsed or refractory chronic lymphocytic leukemia. N Engl J Med. 2018;378(12):110720 Phase 3 randomised trial in relapsed/refractory CLL that showed venetoclax and rituximab had a higher response rate, overall survial and propotion of patient wih undectable minimal residual disease compared with bendamustine and rituximab.

69. Blombery P, Anderson MA, Gong JN, Thijssen R, Birkinshaw RW, Thompson ER, et al. Acquisition of the recurrent Gly101 Val mutation in BCL2 confers resistance to venetoclax in patients with progressive chronic lymphocytic leukemia. Cancer Discov. 2019;9(3): 342-53.

70. Bojarczuk K, Sasi BK, Gobessi S, Innocenti I, Pozzato G, Laurenti $\mathrm{L}$, et al. BCR signaling inhibitors differ in their ability to overcome Mcl-1-mediated resistance of CLL B cells to ABT-199. Blood. 2016;127(25):3192-201.

71. DiNardo CD, Pratz K, Pullarkat V, Jonas BA, Arellano M, Becker PS, et al. Venetoclax combined with decitabine or azacitidine in treatment-naive, elderly patients with acute myeloid leukemia. Blood. 2019;133(1):7-17.

72. Ibrutinib as early therapy in chronic lymphocytic leukemia (CLL) Full Text View - ClinicalTrials.gov [Internet]. [cited 2019 Nov 17]. Available from: https://clinicaltrials.gov/ct2/show/NCT03207555

73.• Jain N, Keating M, Thompson P, Ferrajoli A, Burger J, Borthakur $\mathrm{G}$, et al. Ibrutinib and venetoclax for first-line treatment of CLL. N Engl J Med. 2019;380(22):2095-103 Phase 2 study showing combined ibrutinib and venetoclax in older untreated patients with high-risk CLL results in $62 \%$ having undetetable minimal residual disease.

74. Burger JA, Barr PM, Robak T, Owen C, Ghia P, Tedeschi A, et al. Long-term efficacy and safety of first-line ibrutinib treatment for patients with CLL/SLL: 5 years of follow-up from the phase 3 RESONATE-2 study. Leukemia [cited 2019 Nov 17]. https://doi. org/10.1038/s41375-019-0602-x.

75. Byrd JC, Harrington B, O'Brien S, Jones JA, Schuh A, Devereux S, et al. Acalabrutinib (ACP-196) in relapsed chronic lymphocytic leukemia. N Engl J Med. 2016;374(4):323-32.

76. Dreger P, Schetelig J, Andersen N, Corradini P, Van Gelder M, Gribben J, et al. Managing high-risk CLL during transition to a new treatment era: stem cell transplantation or novel agents? Blood. American Society of Hematology. 2014;124:3841-9.

77. Turtle CJ, Hay KA, Hanafi LA, Li D, Cherian S, Chen X, et al. Durable molecular remissions in chronic lymphocytic leukemia treated with CD19-specific chimeric antigen receptor-modified T cells after failure of ibrutinib. J Clin Oncol. 2017;35(26):3010-20.

78. Porter DL, Levine BL, Kalos M, Bagg A, June CH. Chimeric antigen receptor-modified $\mathrm{T}$ cells in chronic lymphoid leukemia. $\mathrm{N}$ Engl J Med. 2011;365(8):725-33.

Publisher's Note Springer Nature remains neutral with regard to jurisdictional claims in published maps and institutional affiliations. 